\title{
Energy and Shannon entropy for a hydrogen atom confined in a dielectric spherical microcavity
}

\author{
Wang Dehuwa ${ }^{1}$, Jie Zhang ${ }^{1}$, Zhaopeng Sun $^{1}$, Shufang Zhang ${ }^{1}$, and Gang Zhao ${ }^{1}$ \\ ${ }^{1}$ Ludong University
}

July 6, 2021

\begin{abstract}
We calculate the energy and Shannon entropy for a hydrogen atom confined in a dielectric spherical microcavity for the fist time. In contrast to the hydrogen atom in the vacuum microcavity, some unexpected and interesting phenomena appear: First, the turning radius for the bound energy changes from positive to negative depends on the dielectric constant of the spherical microcavity sensitively. With the increase of the relative dielectric constant, the turning radius gets larger. Second, the dielectric spherical microcavity impacts the rearrangement of the excited state energy, and breaks the energy degeneracy of the excited states. At some given radius, there is energy crossover between different orbital. Third, the dielectric in the spherical microcavity affects the Shannon entropy for the confined hydrogen atom greatly. The Shannon entropy in the vacuum microcavity is the smallest and the Shannon entropy increases with the relative dielectric constant. For smaller size spherical microcavity, the Shannon entropy change is always negative, which suggests that the electron density is localized. With the increase of the radius of the microcavity, the Shannon entropy change becomes positive, and the confinement of the electron density gets delocalized. Our results show that we can control the confining effect of the spherical microcavity on the atom by changing the dielectric. This work can guide the future experimental studies for trapping and manipulating of atoms and molecules in the external environment and has some practical applications in metrology and quantum information processing.
\end{abstract}

\section{Hosted file}

submission to INQC.pdf available at https://authorea.com/users/423993/articles/529182-energyand-shannon-entropy-for-a-hydrogen-atom-confined-in-a-dielectric-spherical-microcavity 